\title{
Homologous locus pairing is a transient, diffusion-mediated process in meiotic prophase
}

\author{
Trent A. C. Newman ${ }^{\mathrm{a}, 1}$, Bruno Beltran ${ }^{\mathrm{b}, 1}$, James M. McGehee ${ }^{\mathrm{a}, 2}$, Daniel Elnatan ${ }^{\mathrm{a}, 2}$, Cori K. Cahoon ${ }^{\mathrm{a}, 2}$, Michael R. Paddy ${ }^{\mathrm{a}}$, \\ Daniel B. Chu ${ }^{a}$, Andrew J. Spakowitz ${ }^{\mathrm{b}, \mathrm{c}, \mathrm{d}, 3}$, and Sean M. Burgess ${ }^{\mathrm{a}, 3}$
}

${ }^{a}$ Department of Molecular and Cellular Biology, University of California, Davis, Davis, California; ${ }^{b}$ Biophysics Program, Stanford University, Stanford, California; ${ }^{\mathrm{c} D e p a r t m e n t}$ of Chemical Engineering, Stanford University, Stanford, California; ${ }^{\mathrm{d} D e p a r t m e n t ~ o f ~ M a t e r i a l s ~ S c i e n c e ~ \& ~ E n g i n e e r i n g, ~ S t a n f o r d ~ U n i v e r s i t y, ~ S t a n f o r d, ~ C a l i f o r n i a ~}$

\begin{abstract}
The pairing of homologous chromosomes in meiosis I is essential for sexual reproduction and is mediated, in part, by the formation and repair of Spo11-induced DNA double strand breaks (DSBs). In budding yeast, each cell receives $\sim 150-200 \mathrm{DSBs}$, yet only a fraction go on to form crossover products. How and why the cell initially coordinates so many interactions along each chromosome is not well understood. Using a fluorescent reporter-operator system (FROS), we measure the kinetics of interacting homologous loci at various stages of meiosis. We find that while tagged loci undergo considerable motion throughout prophase I, they are constrained in how far they can diffuse from their homolog pair. This effective tethering radius decreases over the course of meiosis in a DSB-dependent manner. We develop a theoretical model that captures the biological contributions of centromere attachment to the nuclear envelope, homolog pairing, and nuclear confinement. With this model, we demonstrate that the experimentally observed heterogeneity in single-cell behavior and the effective tethering between loci is captured for two polymers forming randomly-spaced linkages. The small number of connections required to reproduce our data demonstrates that a single linkage site between homologous chromosomes can constrain the movement of loci up to hundreds of kilobases away.
\end{abstract}

homologous chromosome pairing | meiosis $\mid$ tetO/TetR-GFP $\mid$ polymer physics

$\mathrm{M}$ eiosis is a cellular program that creates haploid gametes from diploid parent cells. This chromosome reduction occurs by two chromosome segregation events that follow one round of DNA replication. In meiosis I prophase, homologous chromosomes pair and recombine using homologous recombination before separating at anaphase I. Errors in pairing can lead to chromosome nondisjunction and are a major contributor to birth defects, such as Down syndrome and miscarriages in humans $(1,2)$.

In yeast, the progression of pairing is often measured by monitoring whether individual homologous loci are colocalized. Loci start off colocalized prior to meiotic DNA replication in the G0 state (Fig. 1a). This colocalization, often referred to as pre-meiotic pairing, is disrupted during the course of meiotic S-phase and restored during meiosis prophase I $(3,4,5,6)$ (see Fig. 1a-c and Fig. 1d). While the mechanism that promotes colocalization in premeiotic cells is not well understood, it is known that the inter-homolog linkages that promote colocalization during prophase I depend on the formation and repair of DSBs created by $\operatorname{Spo} 11(7,8,9)$. For any given meiosis, any sequence has the "potential" (albeit not all equally) to experience a DSB (8). However, only 94 DSBs (2-10 per chromosome) go on to form crossovers (10). It is not known if these excess DSBs are necessary to mediate pairing or if the smaller number that go on to form COs are sufficient.

The sequence of colocalization at G0, separation during S-phase, and reestablishment of colocalization in prophase I is supported by data generated using various physical assays (11), including fluores- cence in situ hybridization to measure the spatial proximity of pairs of loci in fixed spread chromosome preparations $(3,12)$, a chromosome collision assay to measure the relative frequency of DNA/DNA contacts between loci using Cre/loxP site-specific recombination (13), chromosome conformation capture $(14,15)$, and one-spot two-spot measurements using fluorescence reporter operator systems (FROS) in living cells $(5,16)$. While each method has its limitations $(17,18)$, an overall pattern emerges. Full-length homolog juxtaposition seems to rely on a large number of interactions between multiple loci along each chromosome (19).

Existing measurements provide only static snapshots of populations of cells, and it has not been possible to infer whether homologous loci are brought together in the first place by a processive motor or simply via thermal fluctuations. It has been proposed that homologs may undergo many, transient interactions throughout prophase $(3,19)$. However, static measurements are unable to distinguish what fraction of the colocalized homologous pairs are directly interacting, via some form of stable local linkage (as in Fig. 2d), and how many are merely diffusing past each other (as in Fig. 2c).

In order to measure any active forces pulling together homologous loci, and to differentiate between the stably linked and freely diffusing loci, we used FROS-based tags to track pairs of homologous loci that are known to colocalize with high probability. Snapshots of the position of these loci in 3D space over time in individual live cells

\section{Significance Statement}

Meiosis is essential for sexual reproduction, and homologous chromosome pairing is a critical step in this process that must be reliably achieved. We measure the dynamics of homologous loci throughout prophase I of meiosis, demonstrating the transient nature of homolog contacts and heterogeneity in single-cell behavior. We develop a minimal model containing only the basic polymer physics of DNA but is sufficient to reproduce the observed behavior. We show that it only takes a handful of homologous linkages per chromosome to facilitate pairing, demonstrating that a single tethered locus can drastically restrict the diffusion of DNA tens to hundreds of kilobases away. These results demonstrate the central role of random diffusion and polymer physics in facilitating chromosome pairing in meiosis.

T.A.C.N., B.B., J.M.M., C.K.C., D.E., M.R.P., D.B.C., A.J.S., and S.M.B. designed the research T.A.C.N., B.B., J.M.M., C.K.C., D.E., A.J.S., and D.B.C. collected data; T.A.C.N., B.B., J.M.M. C.K.C., D.E., A.J.S., and S.M.B. analyzed the data; T.A.C.N., B.B., J.M.M., A.J.S., and S.M.B. wrote the paper.

The authors declare no conflict of interest.

${ }^{1}$ T.A.C.N. and B.B. contributed equally to this work. ${ }^{2}$ J.M.M., D.E., and C.K.C. contributed equally to this work.

${ }^{3}$ To whom correspondence should be addressed. E-mail for A.J.S.: ajspakow@stanford.edu. Email for S.M.B.: smburgess@ucdavis.edu. 
(a)

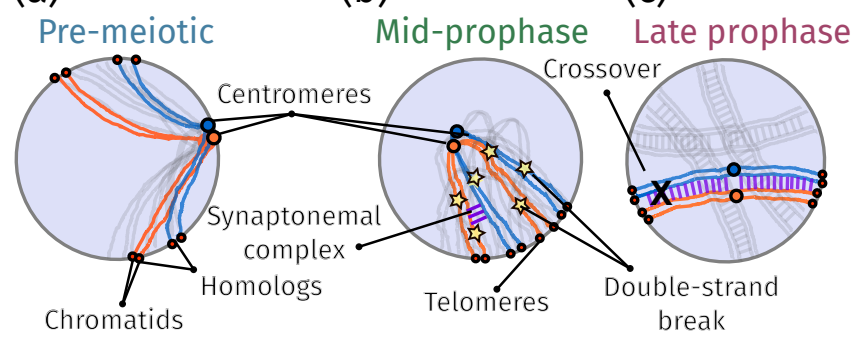

(d)

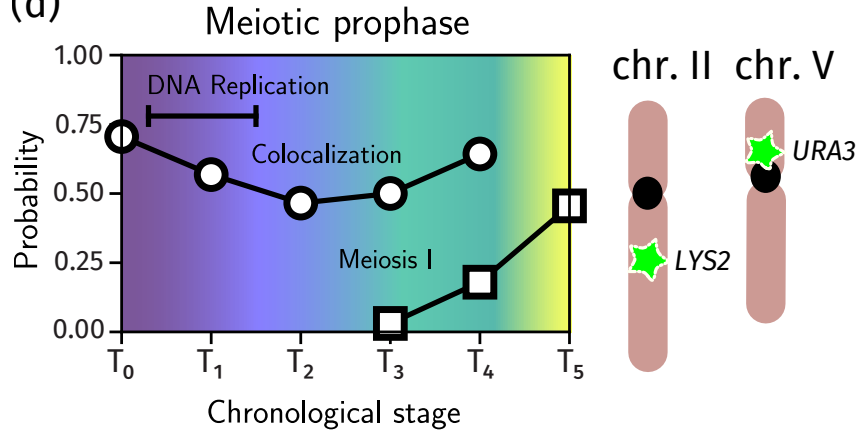

Fig. 1. A schematic of the relative timing of the chromosome events of meiosis in SK1 strains of budding yeast $(3,4,5,13,20,21,22)$. (a) Chromosomes in pre-meiotic cells arrested in G0 are in the Rabl configuration with centromeres tethered to the nuclear periphery (23) and homologous chromosomes form loose associations $(3,24)$ (b) Early- to mid-prophase is marked by dissolution of the Rabl configuration, DSB formation, initiation of synapsis $(20,25)$, and reorientation to form a bouquet where telomeres cluster to one side of the nucleus (26). (c) Late prophase is marked by the end-to-end alignment of homologs by the synaptonemal complex. (d) Fraction cells over time that demonstrate colocalization of the URA3 locus and completion of meiosis I (MI). The x-axis measures the time $T_{i}$ ( $i$ hours) after induction of sporulation that the cells in question were prepared for imaging. Pre-meiotic colocalization is lost during DNA replication and is restored during meiotic prophase, culminating in the full-length alignment of homologs joined by the synaptonemal complex (SC) Soon afterwards, cells begin to complete meiosis I (MI). (e) The relative positions along the chromosome of our tagged loci are shown. These loci were chosen to probe the dependence of colocalization on centromere proximity. were collected in vivo in the G0 state prior to DNA replication and in cells transiting through prophase I to anaphase I.

Any mechanism to bring together or stabilize the loci in vivo must either utilize or overcome the thermal motion of the DNA polymer. Thus, we compare our data to a minimal model, designed to capture only the basic, well-established physical properties of meiotic chromatin. We then modify this baseline model to include linkages between randomly-chosen homologous sites. While a model with randomly chosen linkage sites only rarely produces a configuration where the labeled loci are directly linked, distal linkages result in the measured loci being effectively tethered together (see Fig. 2c-d) by a spring-like force. Because a small handful of DSBs per chromosome are known to mature into stable CO products (10) and will contribute such a spring-like tethering force, any measurement of the force between homologous loci must acknowledge this effect.

Comparing the experimental data to our polymer model suggests that the small number of linkages caused by COs (27) (or recombination intermediates that will become COs), are sufficient to explain the ubiquitous colocalization we observe in vivo. Even though the nearest linkage site is often tens or hundreds of kilobases away from our tagged loci, our theory is still able to reproduce the dynamics of the tagged loci throughout meiosis, suggesting that these loci are not directly interacting, but are merely tethered together indirectly by distal homologous interactions (as in Fig. 2b-c). Our results suggest a handful of bona fide Spo11-dependent linkages per chromosome are sufficient to drive end-to-end homolog juxtaposition.

\section{Results}

Live imaging reveals physical tethering between homologous loci. Our study used yeast strains containing chromosomes carrying FROS tags comprised of chromosomally-integrated tet operator arrays of 112 repeats bound by fluorescent TetR-GFP protein (5). Operators were inserted at either the URA3 locus-which is on the short arm of chr. V near the centromere, or the LYS2 locus-which is in the center of the long arm of chr. II (see Fig. 1).

Cells were cultured for synchronized progression through meiotic prophase as described in Ref. (28). Briefly, cells were grown in YP media containing acetate for arrest in G0. Thereafter, cells were transferred to sporulation medium and aliquots of cells were removed from the culture every hour $\left(T_{M}=T_{0}, T_{1}, \ldots\right)$ and imaged over a 25 minute period at 30 second intervals $\left(t_{i}=0,30, \ldots, 1500\right)$. Following extensive quality control (see Supplementary Information), the positions $\vec{r}_{1}\left(t_{i}\right)$ and $\vec{r}_{2}\left(t_{i}\right)$ of the two fluorescent foci (or the single paired focus) was determined for slides with at least 10 "ok" cells present, as seen in Fig. 3.

Since Spo11-dependent homolog colocalization begins shortly after 3 hours post transfer to sporulation media, we first verified that our trajectories exhibited evidence of tethering between the two homologous loci at $T_{3}$. This was done by comparing the height of the plateau of the mean-square displacement (MSD) curves of individual loci to the mean-square change in distance (MSCD) curves of those same loci. Following (29), we define the MSCD to be the mean-squared change of the vector connecting the two loci, $\Delta \vec{r}=$ $\vec{r}_{2}\left(t_{i}\right)-\vec{r}_{1}\left(t_{i}\right)$. For unlinked loci, we would expect the MSD and MSCD curves to plateau to a comparable value (approximately the square of the confinement radius). Therefore, a MSCD curve which plateaus to a lower level than the MSD curve is indicative of some level of linkage between the two loci. Supplementary Information (Fig. S2) provides the comparison between MSD and MSCD for the URA3 and LYS2 loci, confirming the MSCD curves are substantially smaller than the MSD values.

Figure 4 shows time-averaged, single-cell MSCDs for a random subsample of cells from a single movie of URA3 at $T_{5}$. We compute the time average for a single trajectory as

$$
\left\langle\Delta \vec{r}^{2}(t)\right\rangle_{\mathrm{ta}}=\left\langle(\Delta \vec{r}(\tau+t)-\Delta \vec{r}(\tau))^{2}\right\rangle_{\tau},
$$

where $\langle\cdot\rangle_{\tau}$ indicates the averaging is performed over all possible values of $\tau$. Because our fluorescent tags are a single color, whenever

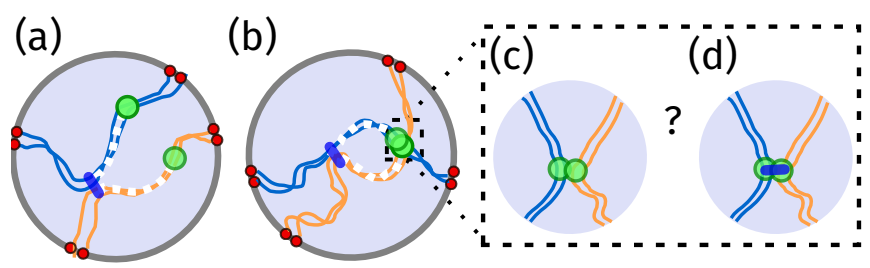

Fig. 2. Schematic illustrating our definition of "tether" and "linkage". (a) An example of a distal linkage (blue), far upstream of the actual tagged locus (green). The length of DNA highlighted in white is the tether length. The longer the tether, the weaker the spring force pulling together the tagged loci. In this example, while the loci are tethered together, they happen to not be colocalized. (b) Here, the two tagged loci are colocalized. However, their colocalization can occur either because the loci have transiently diffused into close spatial proximity (c) or because a linkage has formed at or near the tagged loci (d). 
bioRxiv preprint doi: https://doi.org/10.1101/2021.04.23.440859; this version posted April 24, 2021. The copyright holder for this preprint (which was not certified by peer review) is the author/funder, who has granted bioRxiv a license to display the preprint in perpetuity. It is made available under aCC-BY-NC-ND 4.0 International license.

(a) Separated

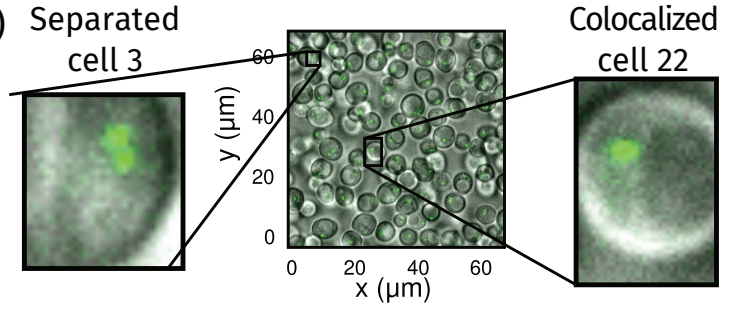

(b)

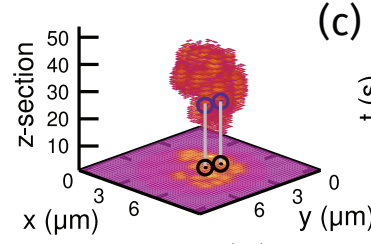

(c)

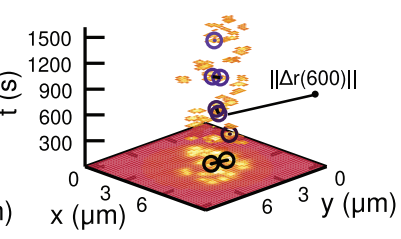

(d) Persistently (e)
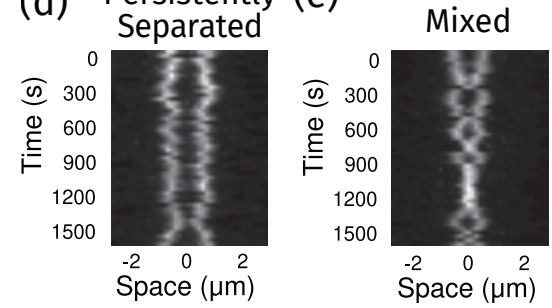

(f) Always

(g)

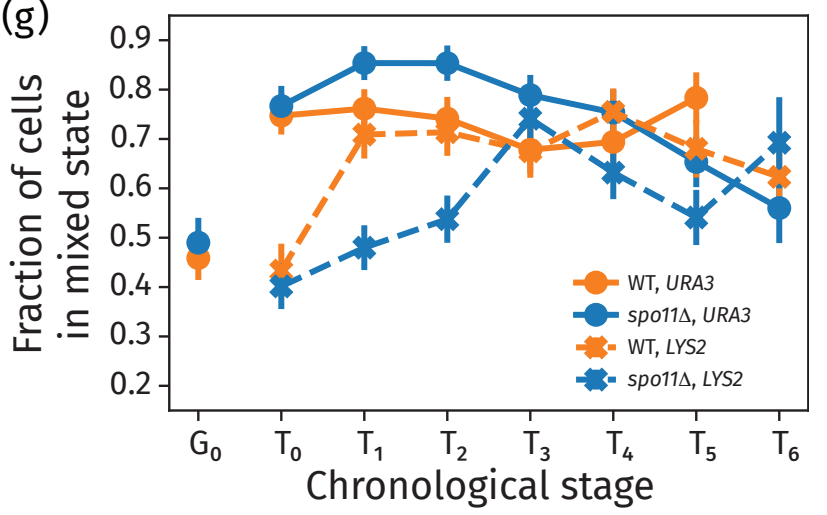

Fig. 3. (a) A typical field of cells, highlighting example cells showing either two spots (left) or one spot (right). (b-c) Maximum intensity projections (MIPs) of the relative positions of fluorescent foci at $30 \mathrm{~s}$ intervals. In (b), the vertical axis corresponds to a $z$-stack (with step size $2 / 15 \mu \mathrm{m}$. For each $\mathrm{x}$ and $\mathrm{y}$ coordinate, the maximum value over all time points for that $z$-stack is shown. In (c), the vertical axis represents time (t, in seconds), and the projection is instead performed over z-stacks. The positions of the loci and the distance between them is highlighted for select time points. (d-f) kymographs showing the distance between the loci in a single cell over the 25 minute imaging period. Each horizontal slice in the kymograph shows the fluorescence intensity along the line joining the centers of the two loci in a single frame. Example of cells where the loci are separated (d), or colocalize (f) for every frame. The cell shown in (e) undergoes several transitions between the two states. (g) Fraction of cells in the mixed state versus chronological time through meiosis for the URA3 and LYS2 loci in wild-type and spo11 $\Delta$ cells. Plot was made from aggregating all available data for each meiotic stage. The error is the standard error of the mean with the sample count set to the number of trajectories (Supp. File 2). The error is the standard error of the mean with the sample count set to the number of trajectories. the loci are within $\approx 250 \mathrm{~nm}$ of each other, their locations are indistinguishable due to overlap of their respective point spread functions. Such time points were omitted from all MSCD calculations, meaning that we are explicitly computing the dynamics from movie frames where the loci are non-overlapping. The Supplementary Information (Fig. S3 for URA3 and Fig. S4 for LYS2) provides plots of the singlecell MSCDs for times $T_{0}$ to $T_{5}$ for wild-type and spo11 $\Delta$ strains.

The top plot of Fig. 4a shows results from wild-type cells, and the bottom plot (b) shows the behavior of spo11 $1 \Delta$ cells. These plots show results from 25 randomly selected cells (light) along with 5 randomly selected cells (bold) to demonstrate the cell-to-cell heterogeneity and individual-cell behaviors. The trajectories exhibit a combination of power-law transport (MSCD $\sim t^{b}$ ) and confined motion (constant MSCD). To clearly demonstrate this behavior, the Supplementary Information (Figs. S5 and S6) provides an analysis of this behavior at $T_{0}$ for both URA3 and LYS2 loci in the wild-type strain. This analysis includes a fit of each single-cell MSCD to a function $M S C D=\min \left(A t^{B}, C\right)$, which exhibits an initial power-law behavior followed by a plateau. From this analysis, the distribution of values of the power-law slope $B$ ranges from about zero to 0.5 , with an average value of $B=0.24$ (see Supplementary Information for details). Figure 4 includes power-law scaling behaviors associated with confined motion (zero slope) and the experimentally determined power-law scaling (slope $B=0.24$ ) as guides.

The MSCD behaviors of wild-type (a) and $\operatorname{spol1\Delta }$ (b) at $T_{5}$ show distinct differences that reflect their underlying biological states. At this late stage of prophase I, we anticipate that most cells are no longer in the Rabl configuration. The spol1 $\Delta$ cells show a clustering of the MSCD plateau between $1 \mu \mathrm{m}^{2}$ and $2 \mu \mathrm{m}^{2}$, which we associate with confined motion within the nuclear environment. Notably, several individual cells in Fig. 4b exhibit a significantly lower MSCD plateau, which are likely due to the rare cases of cells remaining in the Rabl configuration at $T_{5}$ or cells where centromeres are attached to spindle
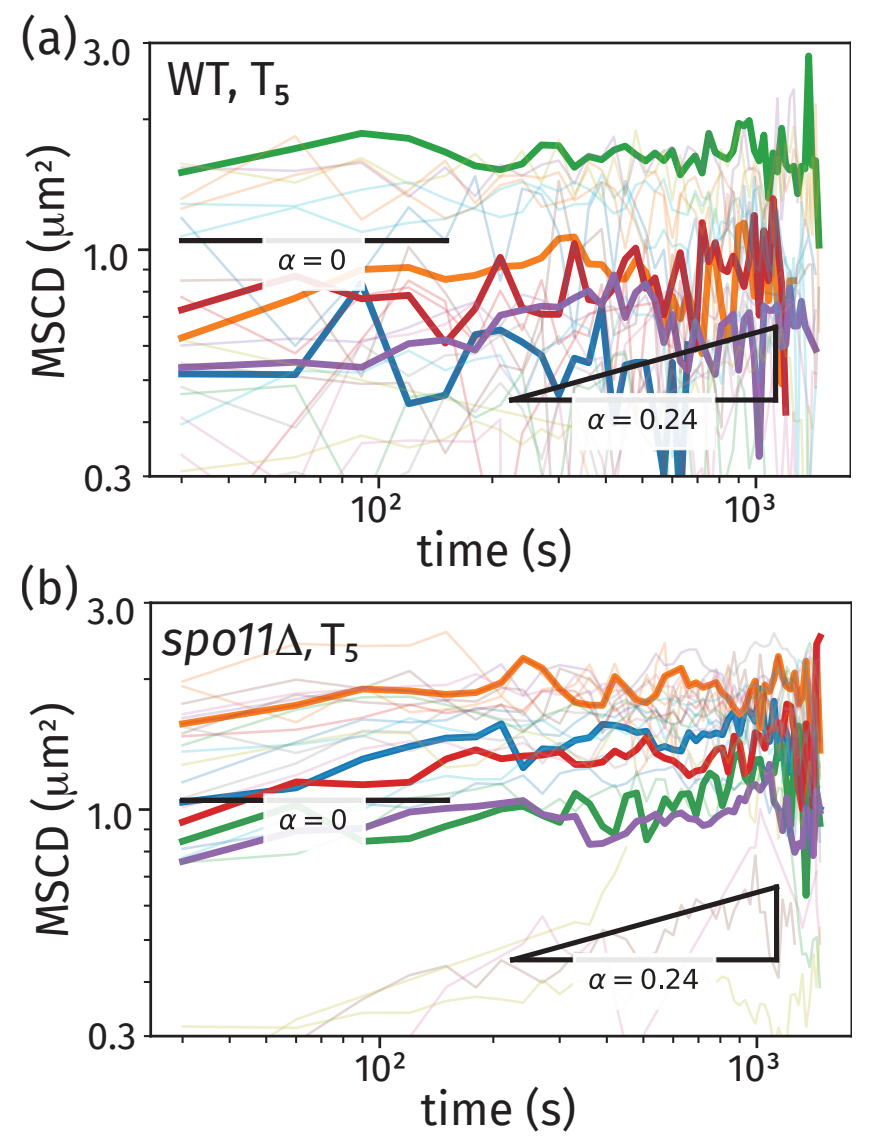

Fig. 4. Single-cell MSCDs for URA3 trajectories at $T_{5}$. These plots show results from 25 randomly selected cells (light) along with 5 randomly selected cells (bold) for wild-type cells (a) and spo11 $\Delta$ cells (b). Each plot includes two power-law scaling behaviors associated with confined motion (slope $\alpha=0$ ) and unconfined polymer motion (slope $\alpha=0.5$ ). 
fibers and about to go through anaphase. The wild-type cells in Fig. 4a show a much larger degree of heterogeneity in MSCD behavior. We proceed to interpret this heterogeneity based on the physical constraints associated with the progression of linkages between the homologous chromosomes throughout prophase I.

\section{Tethering of homologous loci through random linkages can recreate the range of confinement observed experimentally.} Many of the single-cell trajectories in Fig. 4 exhibit confined motion, indicated by the MSCD exhibiting a long-time plateau. The heterogeneity in these plateau values suggest cell-to-cell variability in the dominant contributions to the physical confinement. We identify three major contributors that confine the relative motion of the homologous chromosomes: confinement within the nucleus (1), centromere linkage for cells in the Rabl configuration (2), and linkages between the homologous chromosomes as prophase I progresses (3). We establish a theoretical model of homolog pairing to interpret the experimentally observed behavior with the goal of predicting the impact of these three sources of confinement on chromosome motion during prophase I.

Previous work demonstrates that chromosomal behaviors in living cells, including bacteria $(30,31,32)$, mammalian cells (33), and yeast nuclei $(32,34,35,36)$, are captured by polymer-physics models These works are generally based on the Rouse model (37). In this model, the polymer chain is represented as a linear chain of beads connected by springs, and the motion is driven by random Brownian forces. Several treatments of in vivo dynamics extend the Rouse model to include the influence of viscoelasticity, which we identify as the viscoelastic Rouse model) (30, 31, 32, 32, 33), leading to a significant reduction in the power-law scaling of various metrics (e.g MSD, MSCD, and the velocity autocorrelation function).

The original Rouse model exhibits a monomer MSD with powerlaw scaling of $t^{1 / 2}$, and the viscoelastic Rouse model for a fluid with scaling exponent $\alpha$ (i.e. particle motion exhibits MSD $\sim t^{\alpha}$ ) leads to a monomer MSD with scaling MSCD $\sim t^{\alpha / 2}$. Given the average power-law scaling for our experimental MSCDs having a scaling $B=0.24$, our results are consistent with a viscoelastic Rouse model with $\alpha=2 B=0.48$.

We develop a polymer-physics model of homologous chromosomes that extends the viscoelastic Rouse polymer by adding several key physical contributions. First, we confine two Rouse polymers within a sphere of radius $a$, representing the nuclear confinement. Second, we link the two polymers at the centromere position (chosen appropriately for the specific chromosome being modeled), if the cell is in the Rabl configuration. Third, we model the progression of homolog pairing by adding linkages between the two polymers with increasing average number as pairing progresses. Our model therefore has the following physical parameters: the Kuhn length $b$ of the polymer chains, the spherical radius $a$, the rate constant for transitioning from the Rabl configuration $k_{\text {Rabl }}$, the average number of linkages $\mu$ (varies with time after sporulation), and the diffusion constant $D_{0}$ for polymer segmental motion. The polymer lengths and segmental positions of the tracked loci and centromeres are determined from the genomic properties.

Experimental behavior under various conditions permits us to isolate and determine individual physical parameters in our model. Here, we provide an overview of the procedure used to determine these parameters. The behavior of the MSCD at $T_{0}$ (just after induction of sporulation) is dominated by the centromere linkage for the URA3 locus on chromosome $\mathrm{V}$ due to its close proximity to the centromere. We predict the MSCD plateau at time $T_{0}$ to be $\operatorname{MSCD}_{\infty}\left(T_{0}\right)$ based on the approach to its stable asymptotic value. Using our model applied to chromosome $\mathrm{V}$ in the Rabl configuration, we predict the plateau in the MSCD versus Kuhn length. This analysis is used to determine the Kuhn length to be $b=250 \mathrm{~nm}$.

As the cells progress through prophase I, we assume the change in the MSCD of the spol1 1 strain arises from progressive transition from the Rabl configuration. We evaluate the MSCD plateau at each time from $T_{0}$ to $T_{6}$. We then fit this data to a function of the form $\mathrm{MSCD}_{\infty}=$ $\operatorname{MSCD}_{\infty}\left(T_{0}\right) \exp \left(-k_{\text {rabl }} t\right)+\operatorname{MSCD}_{\infty}\left(T_{\infty}\right)\left[1-\exp \left(-k_{\text {rabl }} t\right)\right]$, where $k_{\text {rabl }}$ is the rate constant for transition from the Rabl configuration and $\operatorname{MSCD}_{\infty}\left(T_{\infty}\right)$ is the MSCD plateau value at long time (i.e. when all cells transition out of the Rabl configuration). Note, $\operatorname{MSCD}_{\infty}\left(T_{0}\right)$ is uniquely determined from the $T_{0}$ MSCD plateau. From this analysis, we determine $k_{\mathrm{Rabl}}=0.605 \mathrm{~h}^{-1}$, resulting in an average time for centromere detachment of $1.65 \mathrm{~h}$ (between $T_{1}$ and $T_{2}$ ).

From the fitted value of $\operatorname{MSCD}_{\infty}\left(T_{\infty}\right)=1.74 \mu \mathrm{m}^{2}$, we model the MSCD plateau using our theoretical model of two flexible polymers confined within a sphere of radius $a$ with their ends attached to the sphere surface (see Supplementary Information for details). Using this model, we determine the best fit sphere radius to be $a=1.59 \mu \mathrm{m}$.

We then use the MSCD plateau values from the wild type strain for $U R A 3$ to determine the mean number of linkages throughout prophase I to be $\mu=0.08$ at $T_{3}, \mu=1.27$ at $T_{4}$, and $\mu=3.36$ at $T_{5}$. We predict the number of linkages between $T_{0}$ and $T_{3}$ to be negligible, and the behavior is dominated by centromere linkage during this early stage of prophase I. Similar analyses for the LYS2 locus yields the mean number of linkages at $T_{5}$ to be $\mu=1.27$, and $\mu=2.06$ at $T_{6}$ (with $\mu=0$ at earlier times).

Figure 5 shows theoretical predictions for the MSCD for simulated "cells" that are generated by adding a Poisson-distributed number of "linkage sites" located at random positions along the homologous chromosomes. Figure 5a shows 5 linkage diagrams for simulated "cells", where the blue sticks identify randomly selected linkages. These five "cells" coincide with the five bold MSCD curves in Fig. 5b. In addition, Fig. 5 b shows predictions for 25 simulated "cells" as light curves (same number of trajectories as presented in Fig. 4), providing a picture of both the individual "cell" behavior and the distribution within the ensemble. These smooth MSCD curves generated by our theory predict the behavior from a time average over random trajectories (i.e. driven by Brownian motion) for the fixed linkages of each "cell".

The two copies of our tagged loci are connected by an effective tether whose length is dictated by the distance to the nearest linkage sites, which we highlight in Fig. 5a using bold white for the nearest linkage and thin white for the next-nearest linkage (if applicable). If the tagged locus has a linkage on only one side (e.g. cells 1 and 4 in Fig. 5a), the tagged loci are tethered together by a linear chain. If there are linkage sites on both sides of the tagged locus (e.g. cells 2, 3, and 5 in Fig. 5a), the tagged loci are isolated within an effective "ring" polymer. Assuming these topologies are fixed, we analytically compute the MSCD of the tagged loci by treating them as beads connected by Rouse polymers of appropriate lengths and topology (see Supplementary Information for details on our analytical theory for the MSCD of linear and ring polymers).

Figure 5b shows analytical MSCD curves for the 5 "cells" shown schematically in Fig. 5a. The effective tethering radii (MSCD plateau heights) for the randomly linked chromosomes span a similar range as the wild-type data in Fig. 4a. This heterogeneity in predicted behavior arises from variability in the location of the nearest linkage. Instances where a randomly positioned linkage is in close genomic proximity to the tagged locus (e.g. cell 4) result in low values of the MSCD plateau. Variability in the distance to the nearest linkage causes the MSCD 
(a)

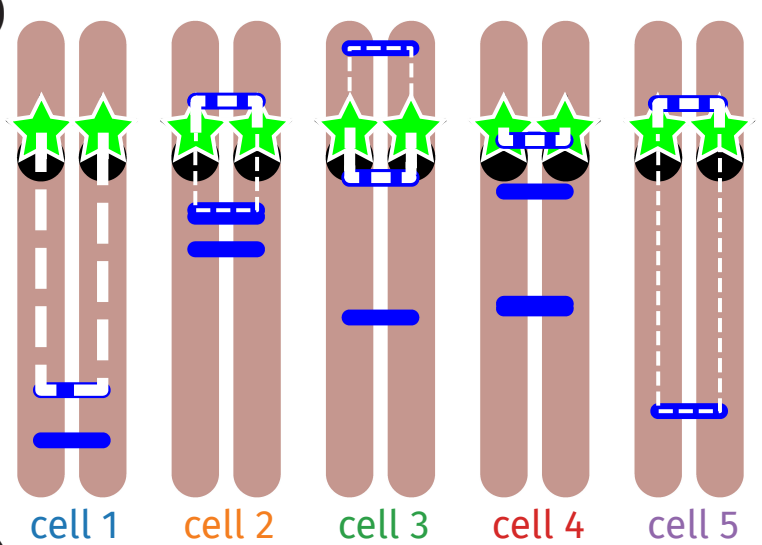

(b)

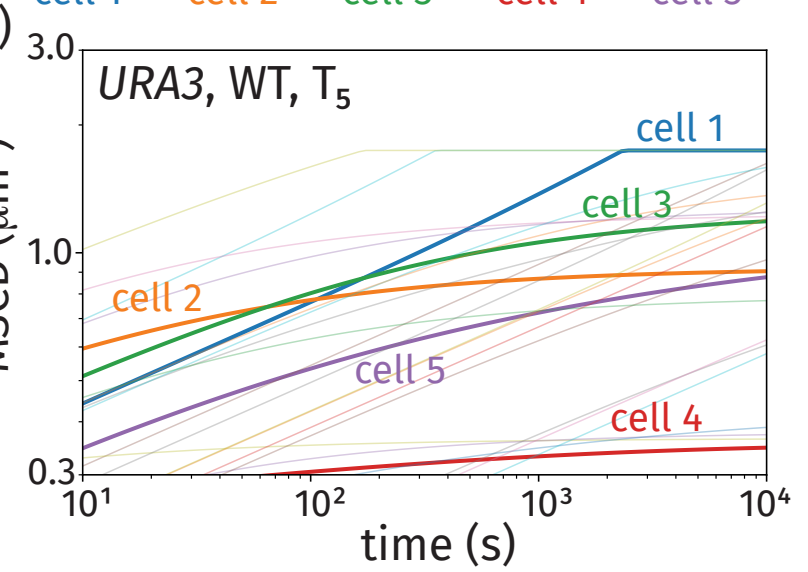

Fig. 5. Theoretical predictions for the MSCD based on our random-link model for homolog pairing coincident with URA3 trajectories at $T_{5}$. Five individual cell linkage diagrams (a) result in the five bold MSCD curves the plot (b). The MSCD plot shows 25 additional realizations (light) to demonstrate the heterogeneity in the MSCD behavior.

curves to vary in their magnitude, and there are instances where the nearest linkage is sufficiently far that the nuclear confinement dictates the MSCD plateau, as in cell 1 in Fig. 5. Prior to the plateau, each MSCD curve in Fig. 5 exhibits a transient power-law scaling of $t^{0.24}$, as dictated by the viscoelastic Rouse model.

Progression of behavior through prophase I dictated by centromere release and linkage formation. The individual-cell MSCDs at $T_{5}$ in Figs. 4 and 5 demonstrate the late-stage behavior, after transition from the Rabl configuration. We now analyze the ensemble-averaged MSCD at each meiotic stage $\left(T_{M}\right)$ to demonstrate how the biophysical contributions to the dynamics evolve over the course of meiosis. This progression is marked by two offsetting events: release of the centromere and formation of Spo11-dependent linkages. We use a dual time-and-ensemble average MSCD, computed as

$$
\left\langle\Delta \vec{r}^{2}(t)\right\rangle_{\mathrm{ens}}=\left\langle\left(\Delta \vec{r}_{j}(\tau+t)-\Delta \vec{r}_{j}(\tau)\right)^{2}\right\rangle_{j, \tau},
$$

where $\Delta \vec{r}_{j}$ refers to the distance between the two loci in the $j$ th cell, and the average is taken over all cells imaged at each $T_{M}$ (across multiple biological replicates).

In Fig. 6a and Fig. 6b, we show the ensemble-average MSCD curves for wild-type and spol1 $1 \Delta$ strains, respectively, for the URA3 loci, and Fig. 6c and Fig. 6d are the corresponding plots for the LYS2 loci. From this experimental data, we fit the subdiffusion coefficients $D_{0}\left(T_{M}\right)$ at each time using results from our theoretical model, which include Rabl transition and progressive linkage formation (based on analyses from the previous section). The values of the fitted subdiffusion coefficient are provided in the Supplementary Information in Fig. S7. We find that the early stage data is better fit by a lower diffusivity, and this diffusivity becomes progressively larger as the cells progress through prophase I. Figure 6 shows results of our theoretical model at each time as the solid curves based on 100,000 realizations of our theoretical "cells" whose individual contributions are demonstrated in Fig. 5. The random Brownian motion from each trajectory and cell-to-cell heterogeneity from linkage positioning is smoothed out from the combination of ensemble and time averaging within the theory. In our determination of the theoretical average, we exclude MSCD values that are below the detection threshold of $0.0625 \mu \mathrm{m}^{2}$ to aid comparison with our experimental results that also have this positive bias.

Figure 6 includes arrows to clarify the progression of behaviors throughout meiosis. Notably, the wild-type results in Fig. 6a and c exhibit a non-monotonic behavior, which contrasts the monotonic behavior in the spol1 $\Delta$ data in Figs. $6 \mathrm{~b}$ and d. At early times, the MSCD is substantially reduced due to two effects: the large fraction of cells in the Rabl configuration and the reduced subdiffusion coefficient at this early stage. The MSCD increases through this early stage as more cells no longer are linked at the centromere and the subdiffusion coefficient progressively increases. This gradual increase is consistent with previous work (4) that reports significant heterogeneity in the time between induction of sporulation and entry into meiosis, despite the use of synchronized cell cultures. As the centromere dissociates from the nuclear envelope in more and more cells_leaving the loci free to diffuse throughout the nucleus - the average plateau level would be expected to rise concomitantly. Furthermore, the increases in the subdiffusion coefficient is consistent with the observation of rapid prophase movement at the telomeres $(38,39,40)$. Notably, the increase in the subdiffusion coefficient is more dramatic for the URA3 locus than the LYS2 locus (see Supplementary Information, Fig. S7), which is likely due to the closer proximity of the URA3 locus to a telomere on chromosome $\mathrm{V}$ than the corresponding distance to a telomere for LYS2 on chromosome II.

At $T_{3}$, around when we expect bona fide homologous recombination to begin, the average confinement radius for the URA3 locus begins to decrease again (see Fig. 6a). Similar behavior is seen for the LYS2 locus in Fig. 6c, but the inversion is first quantifiable at $T_{4}$. In both cases, the MSCD decreases as more linkages are formed between the homologous chromosomes. This reduction in the MSCD and MSCD plateaus is only expected in wild-type cells, as the spol1 $\Delta$ mutants do not form linkages arising from Spo11-induced doublestrand breaks. This is generally true in our experimental data in Figs. $6 \mathrm{~b}$ and $\mathrm{d}$. However, time $T_{5}$ for URA3 locus in the spol1 $\Delta$ mutant (see Fig. 6b) exhibits a reduced MSCD before going back to the terminal MSCD plateau at time $T_{6}$.

To verify that the observed behaviors in Fig. 6 is specific to homologous chromosomes and not simply due to large-scale nuclear compaction, we repeated our analyses in a strain where our FROS tag is integrated in only one homolog of chromosomes V and II at the $U R A 3$ and LYS2 loci. In these cells, the MSCD plateau level instead increases starting at $T_{3}$ (see Supplementary Information), confirming that the confinement we see beginning at $T_{3}$ is specific to homolog pairs.

Homologous interactions remain transient throughout meiosis. Our single-cell measurements permit us to evaluate the kinetics of transient interactions between loci. In Fig. 7, we report the fraction of time the two loci exist in a colocalized state (i.e. their point- 
bioRxiv preprint doi: https://doi.org/10.1101/2021.04.23.440859; this version posted April 24, 2021. The copyright holder for this preprint (which was not certified by peer review) is the author/funder, who has granted bioRxiv a license to display the preprint in perpetuity. It is made available under aCC-BY-NC-ND 4.0 International license.

(a)

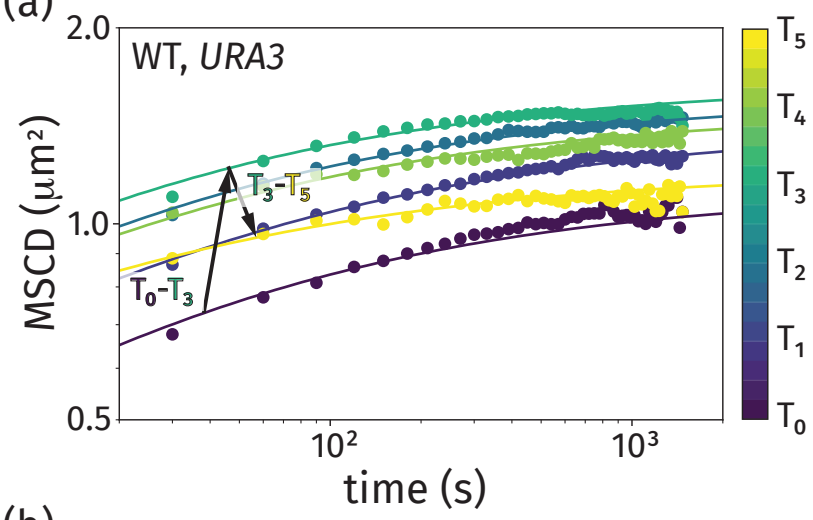

(b)

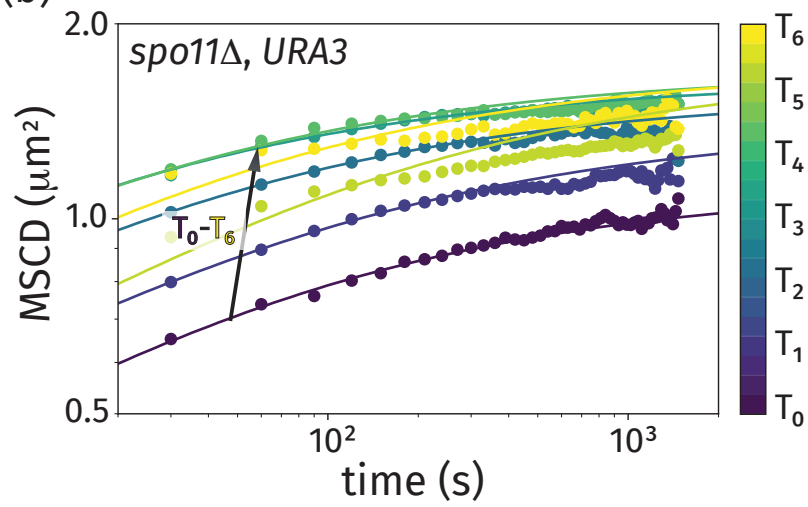

(c)

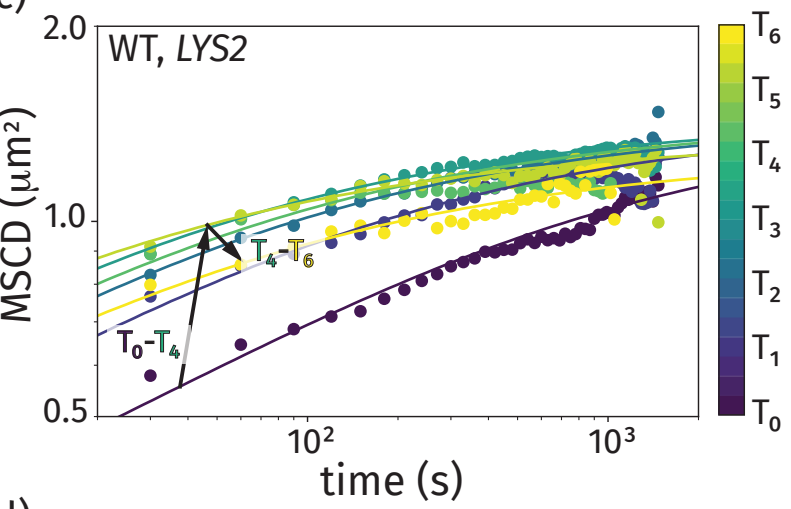

(d)

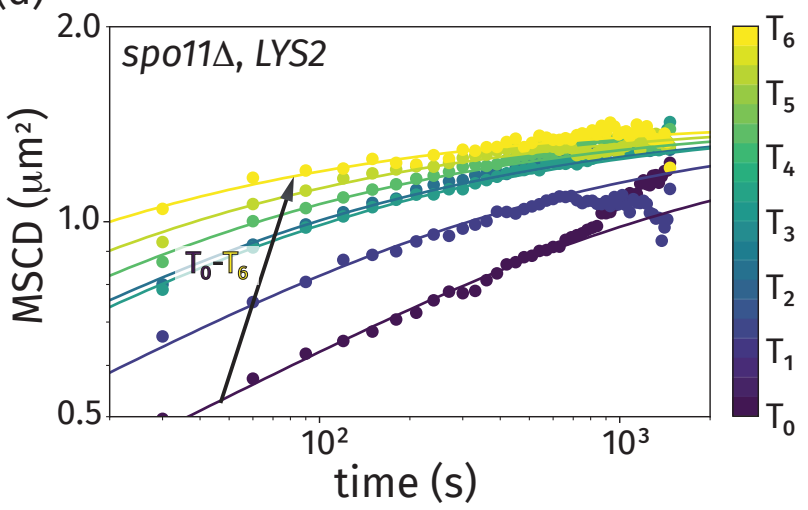

Fig. 6. Time-and-ensemble averaged MSCDs at different times after induction of sporulation, for wild-type strain tagged at the URA3 locus (a), spo11 $\triangle$ strain tagged at the URA3 locus (b), wild-type strain tagged at the LYS2 locus (c), and spo11 $\Delta$ strain tagged at the LYS2 locus (d). Theoretical predictions from our model are included for the fitted diffusivities.

spread functions are not distinguishable with a separation of less than $250 \mathrm{~nm}$ ), averaged over all cells imaged and over all frames of each movie. In spol1 $1 \Delta$ mutants (both for the URA3 and LYS2 loci), the fraction of time colocalized continues to decrease over time. However the wild-type cells exhibit a non-monotonic trend in the fraction of time colocalized, as the loci spend more time together during the late stage of prophase I (times $T_{3}$ to $T_{6}$ ). As previously reported by others $(5,41)$, our results exhibit a fraction of one-spot cells that increases during this late state (but never reached 100\%). Due to the static nature of this metric, previous studies have been unable to distinguish between an increased frequency of transient colocalization on the one hand and the formation of stable interactions in a fraction of the cells on the other.

Using the dynamic information in our measurements, we further classified entire trajectories as being persistently separated-i.e. never forming - and persistently colocalized-remaining in contact throughout the movie. Moreover, by observing trajectories over time, we identified a third category of "mixed" trajectories, where the cell was observed to transition in or out of a colocalized state during the 25 minute period. These three states are easily distinguishable in locus-separation kymographs (see Fig. 3, and Supplementary Information Fig. S15-19). From the "mixed" trajectories, we determine the distribution of dwell times in the colocalized and separated states.

Figure 8 shows the distribution of dwell times for the loci to be in the colocalized and separated states for the LYS2 locus (see Supplementary Information, Fig. S9 for corresponding plots for URA3). The experimental data exists in the colocalized state if the tagged loci are within $250 \mathrm{~nm}$ of each other; otherwise, the loci are in the separated state. This data demonstrates the transient nature of the colocalization of the loci throughout the observation for both the wild-type (Figs. 8a and c) and spol1 (Figs. $8 \mathrm{~b}$ and d) strains. These plots are shown on a log-log scale, which clearly demonstrates the power-law nature of the dwell time distributions. This behavior is clearly distinguishable from an exponential distribution (red curve in Fig. ??a) that typically arises in reaction processes with a single governing time-constant for the transition. Such power-law distributions arise in diffusion-limited

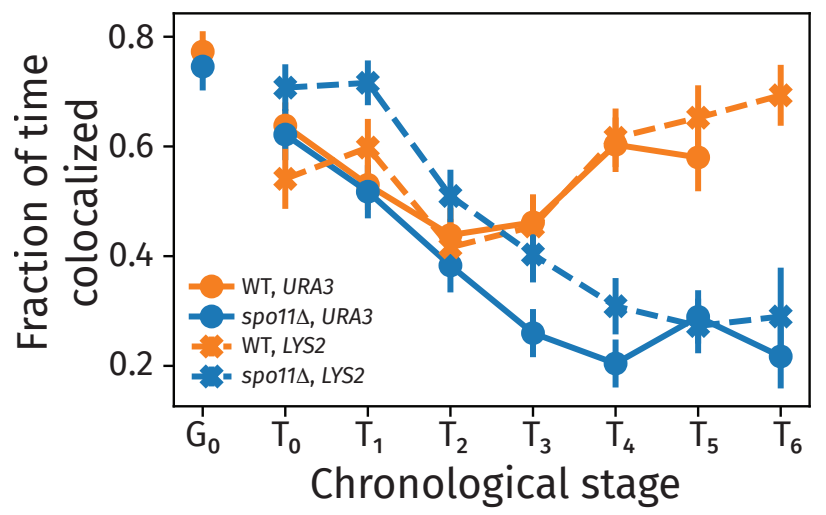

Fig. 7. The fraction of time at each stage of meiosis $\left(T_{M}=T_{0}, T_{1}, \ldots\right)$ that are in a colocalized state for each of the two loci and strains examined. Plot was made from aggregating all available data for each meiotic stage. The error is the standard error of the mean with the sample count set to the number of trajectories (Supp. File 2). 
(a)

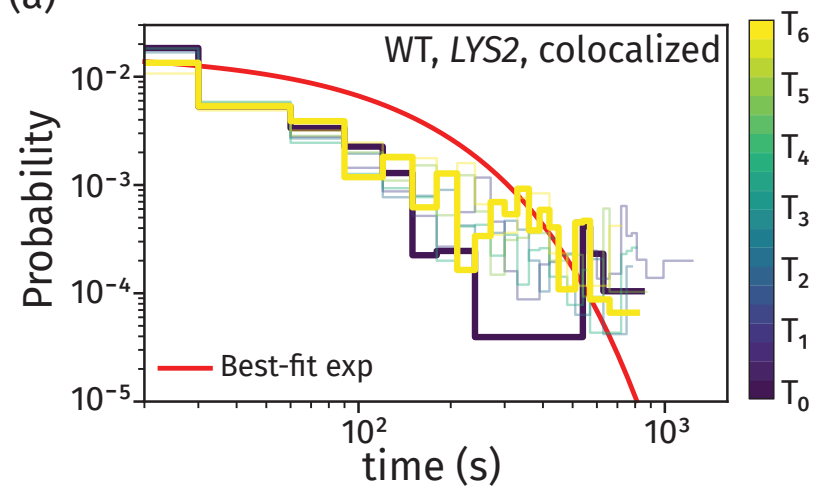

(b)

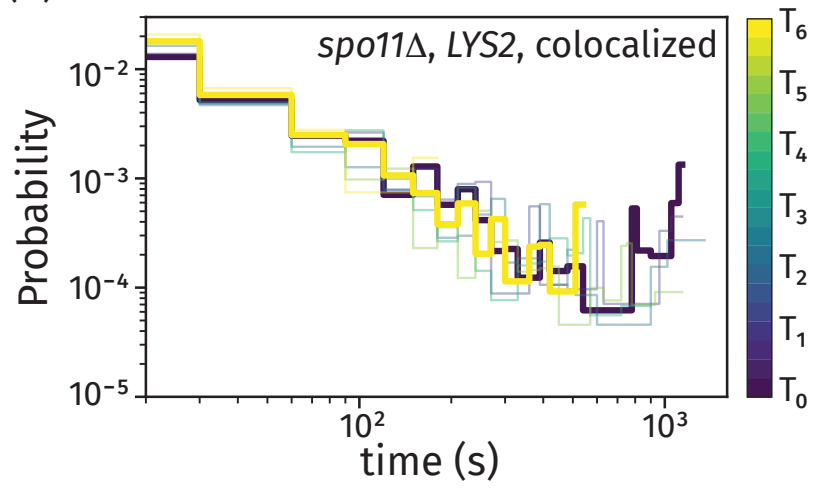

(c)

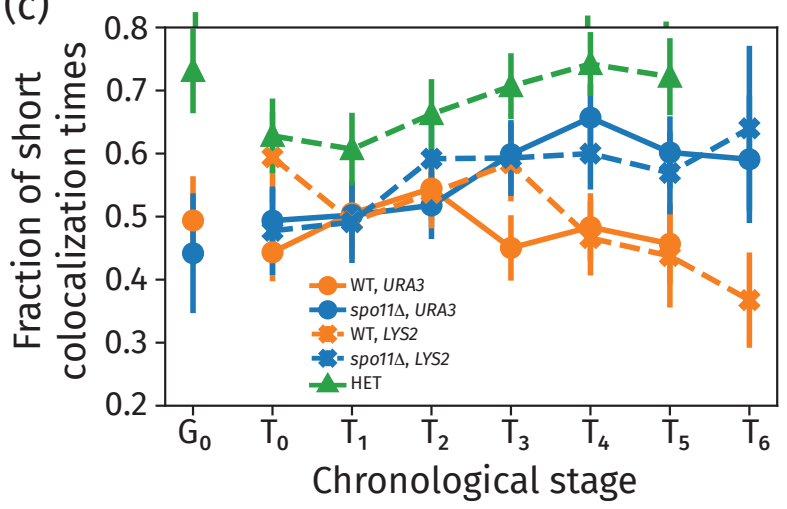

(d)

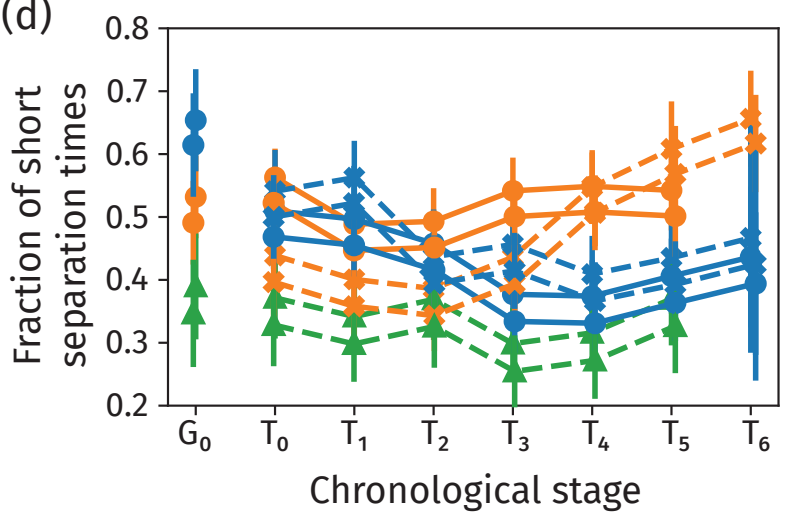

Fig. 8. Histograms of dwell times in the colocalized and separated states for the LYS2 locus. One histogram per stage in meiosis is shown, colored by the time since transfer to

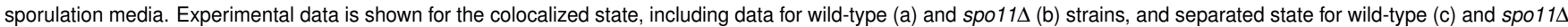
(d) strains. The red curve in (a) shows the best-fit exponential distribution to the data.

intra-chain processes between polymers due to the inherent spectrum of conformational relaxation times (42), which is consistent with the theoretical model presented in this work.

While the general trends in the dwell-time distributions are similar for wild-type and spo11 $\Delta$ strains, we note several important distinctions. The colocalization dwell-time distribution for wild-type cells (Fig. 8a) exhibits a marked progression through meiosis (from $T_{0}$ in purple to $T_{6}$ in yellow) towards favoring longer dwell times in the colocalized state, marked by a long-time tail in the distribution for $T_{6}$. This trend is apparent as a reduced fraction of short colocalization times (i.e. the probability for times less than 30 seconds) over the course of meiosis at the LYS2 and URA3 loci in wild-type cells (Fig. 8c). In contrast, spol1 $\Delta$ cells, and cells with tags on heterologous chromosomes (also see Supplmentary Figure S10), showed a higher fraction of short dwell times later in meiosis (Fig. 8c).

\section{Discussion}

Locus "pairing" is a thermally-dominated process. Earlier studies have used a static "one-spot, two-spot" measurements to analyze the colocalization of individual loci $(5,39)$. In these previous studies, colocalized loci were called "paired", and the DSB-dependence of this pairing led many to speculate that it may be critical for the progression of whole-length homolog pairing $(3,4,19)$. It was demonstrated early on $(3,12,16)$ that a given locus under study will never be paired in every cell, even late in prophase when homologs are synapsed along their lengths. Here, we extend this idea, observing that the vast majority of so-called paired loci are merely in close spatial proximity, and not actually interacting, no matter what stage of prophase we observe. Furthermore, we show that, due to the dynamics of the chromatin polymer, a typical locus will naturally fluctuate into and out of proximity with its homologous partner throughout prophase.

Since our frame rate is $1 / 30 \mathrm{~Hz}$, we cannot rule out the existence of interactions whose effects last less than $30 \mathrm{~s}$, or where the interaction strength is weak enough that it can be drowned out by thermal noise. However, while such interactions may still exist, adding them would (by definition) not affect the output of our model, making it difficult to imagine how such a putative interaction (e.g. repair of DSBs that do not go on to form crossovers) could contribute to the full-length pairing of homologous chromosomes in vivo.

Since the chromatin polymer is thermally fluctuating regardless of cell type, we hypothesize that thermal fluctuations may be a dominant player in driving homolog colocalization in other organisms as well. For example, some authors have observed transient locus pairing in S. pombe (43), Drosophila (44), C. elegans (45) and mouse. It would be interesting to see what fraction of these pairing events can be attributed purely to polymer diffusion.

A pairing process primarily driven by diffusion would also provide a simple explanation for other well-conserved phenomena, such as rapid telomere movement $(38,39,40)$. Instead of pushing or pulling telomeres together, rapid telomere movement need only increase fluctuations along the polymer in order to facilitate pairing $(35,38)$.

The cell-to-cell heterogeneity in dynamic behavior arises from the variability in the timing of biological events (e.g. transition from the Rabl configuration), intrinsic cell-to-cell variability in the diffusivity (46), and the formation of linkages that are randomly positioned along homologous chromosomes. Relating single-cell results (char- 
acterized in Fig. 4) to ensemble-average behavior (shown in Fig. 6) is facilitated by our theoretical model, which captures these various contributions using a minimal description of the linked chromosomal dynamics.

Distal connections can facilitate chromatin organization. The number of linkages required to explain the observed MSCD seen for the URA3 and LYS2 loci at late time points (3.36 and 2.06, respectively) is more consistent with the lower number of crossovers per chromosome compared to the total number of DSBs (47). The measured number of crossovers based on DNA sequencing is $\sim 2-4$ on chromosome $\mathrm{V}$ (on which URA3 resides) and $\sim 6-7$ on chromosome II (on which $L Y S 2$ resides) $(10,48,49)$. Our model predicts the number of linkages at $T_{5}$ for the URA3 locus to be 3.36, which is consistent with the experimentally determined number of crossovers.

Our model prediction of 2.06 linkages from the LYS2 data at $T_{6}$ is below the experimental number of crossovers of $\sim 6-7$ on chromosome II. One potential explanation for the reduced number may be that the dynamics (as determined by the subdiffusion coefficient) is significantly lower for LYS2 than for URA3 (see Supplementary Information Fig. S7 for plot of subdiffusion coefficient).

The distance between linkages predicted by our model for chromosome $\mathrm{V}$ is consistent with the placement of interactions every $\sim 60-80 \mathrm{~kb}$ estimated by Weiner and Kleckner (3). Moreover, it reflects the approximate distance at which crossovers are positioned along the lengths of homolog pairs $(\sim 70-100 \mathrm{~kb})$, which is influenced by crossover interference (50).

The number of linkages required also highlights just how much distant chromosomal junctions can affect the diffusive dynamics of a locus. We hypothesize that other processes that rely on chromosome rearrangement may exploit these same physics. For example, enhancer loop formation has been proposed to be facilitated by TAD formation (51). Our data suggests that, in this case, tracking the loci of interest (e.g. the enhancer/promoter pair) over a long enough time frame should be sufficient to extract their connectivity (e.g. TAD size) on a single-cell level, even if the distal connections joining the loci of interest are hundreds of kilobases downstream.

Heavy-tailed co-localization times are likely rate-limiting for meiotic progression. Our experimentally observed dwell time distributions (Fig. 8) differ drastically from what one would expect if loci were brought into proximity by other means besides polymer diffusion. Suppose, for example, that there was an active mechanism pulling homologous loci together. If the active mechanism was ratelimiting by some chemical step (i.e. kinetics dominated by a single reaction), then we would expect the dwell times to follow an exponential distribution $(52,53)$. While some limiting cases for polymer looping times also produce exponential distributions (42), we instead observe the kind of power-law falloff at long times characteristic of a diffusion-limited process. That is, our distributions are significantly more heavy-tailed than one would expect from a reaction-limited process (shown as the red curve in Fig. 8a).

Our model suggests that the reorganization dynamics are largely driven by random diffusion. Our results also suggest that once any homolog pair does manage to interact, then that initial connection between the chromosomes will greatly facilitate the interaction of other homologous loci. This suggests that homolog pairing might happen via a positive feedback mechanism (such as the one proposed in Refs. $(35,36,54,55))$ wherein each random homologous interaction event decreases the colocalization time for all subsequent homologous interactions, allowing the chromosomes to zipper up significantly faster than would be suggested by the single-homolog colocalization time distribution.

Given how well-aligned the homologous chromosomes are during the G0 phase preceding meiosis (due to the combination of Rabl configuration and DSB-independent interhomolog connections (24)), even a small handful of connections that persist into meiotic prophase I would be enough to drastically reduce the expected colocalization time for the first genuine DSB-mediated homologous connection.

\section{Conclusions}

We show here that the process of homolog pairing in meiosis is more dynamic than expected from previous observations of static "snapshots" of pairing. We found a large degree of heterogeneous behavior by measuring the mean-squared change in distance of tagged chromosome pairs in individual cells verses ensemble averages. A minimal polymer model reproduces the inter-locus dynamics in premeiotic cells where chromosomes are constrained by the Rabl configuration. The model can also reproduce the physical linkages between homolog pairs that are mediated by the formation and repair of Spo11-induced double strand breaks. These findings highlight how coarse-grained modeling of the basic polymer physics driving chromatin motion can be a powerful tool when dealing with complex structural and organizational rearrangements in the nucleus.

\section{Materials and methods}

Time course. All yeast strains used were in the SK1 background and are listed in Supp. Fig. S8. Cell synchronization and meiotic induction was performed as described previously (28). Every hour after transfer to sporulation medium, slides were prepared for imaging according to (56), using silicone isolators (Cat. no. JTR20R-2.0, Grace Bio Labs). All of our image processing code is available at https: //github.com/ucdavis/SeeSpotRun.

Imaging. Imaging was performed on a Marianas real time confocal workstation with mSAC + mSwitcher (3i), using a CSU-X1, microlens enhanced, spinning disk unit (Yokogawa). All imaging was performed in a full enclosure environmental chamber preheated to $30^{\circ} \mathrm{C}$, using a microscope incubator (Okolab). Samples were excited with a LaserStack $488 \mathrm{~nm}$ line (3i), observed using an ALPHA PLAN APO 100X/1.46 OIL objective lens (Zeiss), and photographed using a Cascade QuantEM 512SC camera (Photometrics), with a $0.133 \mu \mathrm{m}$ pixel size. Samples were kept in focus using Definite Focus (Zeiss), capturing up to $41 \mathrm{z}$-sections (as required to acquire the complete sample thickness), with a $0.25 \mu \mathrm{m}$ step size, every $30 \mathrm{~s}$ for 50 time points (a total of $25 \mathrm{~min}$ ). Slidebook v5 (3i) was used to run the time-lapse live-cell imaging and export each plane as a separate 16-bit .tiff file.

Video quality control. Videos were excluded from analysis if the quality was so poor as to affect subsequent analysis, with assessments based on signal to noise, signal bleaching, and drift in the $\mathrm{z}$ and $\mathrm{xy}$ dimensions (Supp. Fig. S9a-c). If drift occurred only at the start or end of the video, and was sufficient to affect image segmentation, then the problematic frames were trimmed from the video. Manual cell segmentation, was performed from a zt-MIP (maximum intensity projection, over the $\mathrm{z}$ and $t$ dimensions) using dist3D_gui.m, while referring back to the z-MIP video, ignoring overlapping cells and those at the edge of the field of view. Qualitative observations of cell quality were made by referring to the z-MIP video and the position of each cropped cell. Only cells deemed "okay" (Supp. Fig. S9d-j) were 
bioRxiv preprint doi: https://doi.org/10.1101/2021.04.23.440859; this version posted April 24, 2021. The copyright holder for this preprint (which was not certified by peer review) is the author/funder, who has granted bioRxiv a license to display the preprint in perpetuity. It is made available under aCC-BY-NC-ND 4.0 International license.

included in the subsequent analysis. For inclusion, videos required twice as many live cells as dead (dead/live $<0.5)$ and $>10$ okay cells.

Spot calling. The position of the fluorescent foci within each cropped cell was detected independently for each time point in the video according to the algorithm described in (57). The raw image intensity data from each cropped cell was filtered with a 3D Gaussian kernel to remove as many noise-related local maxima as possible. Peak localization (runSpotAnalysistest.m) was performed through local maxima detection in 3D using image dilation, followed by curvature measurement, which allowed significant peaks to be identified through a cumulative histogram thresholding method. The computational spot calling was manually confirmed in order to remove obvious errors (Supp. Fig. S10-S11) using conf_gui.m. If the fitting routine failed to find peaks in more than half the time points for any given cell, that cell was omitted from the analysis.

Experiment quality control. Experiments with a very poor overall agreement between computational and manual spot calling, with an average difference between detection methods of greater than $10 \%$ at each meiotic timepoint, were excluded from analysis. The manual analysis was performed by calling cells as having one or two spots based on a visual assessment of a $\mathrm{z}$-MIP, this was done for three time points from each $T_{M}$. Whole experiments were also excluded from the final dataset if the meiotic pairing progression could not be confirmed to exhibit various characteristic properties, such as a single, appropriately timed "nadir". This was typically due to an experiment lacking sufficient $T_{M}$ due to exclusion of individual videos.

Trajectory Analysis. Downstream analysis of the extracted trajectories was performed using a custom Python package (multi_locus_analysis (mla) v.0.0.22, see: https://multi-locus-analysis.readthedocs.io/en/latest/). Dwell times were corrected for finite window effects using the method described in (58). Details of the analysis and code used to make plots can be found in the package documentation.

Analytical Theory. The code used to compute the analytical MSCD curves can also be found in the wlcsim codebase under the wlcsim.analytical.homolog module (for documentation, see https://wlcsim.readthedocs.io). Briefly, the MSCD calculation is broken down into two cases. In the case where the loci are in between two linkage sites, we treat them as being on an isolated ring polymer whose size is chosen to match the effective ring formed by the two homologous segments holding each locus (which are tethered at either end by the linkage site). This effective ring is outlined in white for cells 1 and 4 in Fig. 5. Otherwise, we treat the loci as being on an isolated linear polymer meant to represent the segment of chain running from the end of the first chromosome to one locus, then from that loci to the linkage site, from the linkage site to the other loci, and finally from that loci to the end of the second chromosome. Supplementary Information provides a detailed derivation of the MSCD for these two cases and the value of the plateau MSCD for spherical confined of the polymers.

Statistics. Unless otherwise indicated, variation was measured between experimental replicates for each condition using; Jeffrey's 95\% confidence intervals (CI) for proportion response variables (fraction paired, cell type) or standard error of the mean (SEM) for continuous response variables (distance, MSCD).
Data availability. The raw image data was deposited to the Image Data Resource (http://idr.openmicroscopy.org) under accession number idr0063. The scripts required to reproduce the processed data are available on GitHub[da] (https://github.com/ucdavis/SeeSpotRun); this includes the MATLAB interfaces for spot calling, and the Python scripts for preparing the final xyz position dataset (see Supplementary Dataset 1: finalxyz.csv). The Python module used for downstream analysis also contains the final dataset used in the present study, and can be downloaded from the standard Python repositories by executing pip install multi_loci_analysis.

\section{ACKNOWLEDGMENTS.}

We thank the lab of Angelika Amon for our FROS strains. This work was supported by The National Institutes of Health (NIH), grant: R01GM075119. We thank the Light Microscopy Imaging Facility (Molecular and Cellular Biology, UC, Davis). Financial support for A. J. S. is provided by the National Science Foundation, Physics of Living Systems Program (PHY-1707751). B B. acknowledges funding support from the NSF Graduate Fellowship program (DGE-1656518) and from an NIH training grant (T32GM008294).

1. Antonarakis SE, et al. (1992) The Meiotic Stage of Nondisjunction in Trisomy 21: Determination by Using DNA Polymorphisms. The American Journal of Human Genetics 50:544-550.

2. Nagaoka SI, Hassold TJ, Hunt PA (2012) Human aneuploidy: Mechanisms and new insights into an age-old problem. Nature Reviews Genetics 13(7):493-504.

3. Weiner BM, Kleckner N (1994) Chromosome Pairing via Multiple Interstitial Interactions before and during Meiosis in Yeast. Cell 77:977-991.

4. Cha RS, Weiner BM, Keeney S, Dekker J, Kleckner N (2000) Progression of meiotic DNA replication is modulated by interchromosomal interaction proteins, negatively by Spo11p and positively by Rec8p. Genes \& Development 14:493-503.

5. Brar GA, Hochwagen A, Ee LsS, Amon A (2009) The Multiple Roles of Cohesin in Meiotic Chromosome Morphogenesis and Pairing. Molecular Biology of the Cell 20(3):1030-1047.

6. Brown MS, Zanders S, Alani E (2011) Sustained and rapid chromosome movements are critical for chromosome pairing and meiotic progression in budding yeast. Genetics 188(1):2132.

7. Keeney S, Giroux CN, Kleckner N (1997) Meiosis-Specific DNA Double-Strand Breaks Are Catalyzed by Spo11, a Member of a Widely Conserved Protein Family. Cell 88(3):375-384.

8. Keeney S, Lange J, Mohibullah N (2014) Self-organization of meiotic recombination initiation: general principles and molecular pathways. Annual review of genetics 48:187-214.

9. Zickler D, Kleckner N (2015) Recombination, Pairing, and Synapsis of Homologs during Meiosis. Cold Spring Harbor Perspectives in Biology 7(6):a016626.

10. Krishnaprasad GN, et al. (2015) Variation in Crossover Frequencies Perturb Crossover Assurance Without Affecting Meiotic Chromosome Segregation in Saccharomyces cerevisiae. Genetics 199(2):399-412.

11. Burgess SM (2002) Homologous chromosome associations and nuclear order in meiotic and mitotically dividing cells of budding yeast. Advances in genetics 46:49-90.

12. Loidl J, Klein F, Scherthan $\mathrm{H}$ (1994) Homologous pairing is reduced but not abolished in asynaptic mutants of yeast. The Journal of Cell Biology 125(6):1191-1200.

13. Peoples TL, Dean E, Gonzalez O, Lambourne L, Burgess SM (2002) Close, stable homolog juxtaposition during meiosis in budding yeast is dependent on meiotic recombination, occurs independently of synapsis, and is distinct from DSB-independent pairing contacts. Genes \& Development 16(13):1682-1695.

14. Dekker J, Rippe K, Dekker M, Kleckner N (2002) Capturing Chromosome Conformation. Science 295(5558):1306-1311.

15. Kim S, et al. (2017) The dynamic three-dimensional organization of the diploid yeast genome. elife 6.

16. Lee CY, Conrad MN, Dresser ME (2012) Meiotic Chromosome Pairing Is Promoted by Telomere-Led Chromosome Movements Independent of Bouquet Formation. PLoS Genetics 8(5): 1002730.

17. Fuchs J, Lorenz A, Loidl J (2002) Chromosome associations in budding yeast. Journal of Cell Science 115(6):1213-1220.

18. Mirkin EV, Chang FS, Kleckner N (2014) Protein-Mediated Chromosome Pairing of Repetitive Arrays. Journal of Molecular Biology 426(3):550-557.

19. Kleckner N, Weiner B (1993) Potential Advantages of Unstable Interactions for Pairing of Chromosomes in Meiotic, Somatic, and Premeiotic Cells. Cold Spring Harbor Symposia on Quantitative Biology 58(0):553-565

20. Padmore R, Cao L, Kleckner N (1991) Temporal comparison of recombination and synaptonemal complex formation during meiosis in S. cerevisiae. Cell 66(6):1239-1256.

21. Tesse S, Storlazzi A, Kleckner N, Gargano S, Zickler D (2003) Localization and roles of Ski8p protein in Sordaria meiosis and delineation of three mechanistically distinct steps of meiotic homolog juxtaposition. Proceedings of the National Academy of Sciences 100(22):1286512870.

22. Börner G, Kleckner N, Hunter N (2004) Crossover/Noncrossover Differentiation, Synaptonemal Complex Formation, and Regulatory Surveillance at the Leptotene/Zygotene Transition of Meiosis. Cell 117(1):29-45.

23. Jin QW, Fuchs J, Loidl J (2000) Centromere clustering is a major determinant of yeast interphase nuclear organization. Journal of cell science 113(11):1903-1912.

24. Burgess SM, Kleckner N, Weiner BM (1999) Somatic pairing of homologs in budding yeast: Existence and modulation. Genes \& Development 13(12):1627-1641.

25. Obeso D, Dawson DS (2010) Temporal characterization of homology-independent centromere coupling in meiotic prophase. PloS one 5(4):e10336. 
bioRxiv preprint doi: https://doi.org/10.1101/2021.04.23.440859; this version posted April 24, 2021. The copyright holder for this preprint (which was not certified by peer review) is the author/funder, who has granted bioRxiv a license to display the preprint in perpetuity. It is made available under aCC-BY-NC-ND 4.0 International license.

26. Trelles-Sticken E, Loidl J, Scherthan $\mathrm{H}$ (1999) Bouquet formation in budding yeast: initiation of recombination is not required for meiotic telomere clustering. Journal of Cell Science 112(5):651-658

27. Serrentino ME, Borde V (2012) The spatial regulation of meiotic recombination hotspots: are all dsb hotspots crossover hotspots? Experimental cell research 318(12):1347-1352.

28. Lui D, Burgess SM (2009) Measurement of spatial proximity and accessibility of chromosomal loci in saccharomyces cerevisiae using cre /loxP site-specific recombination in Meiosis Volume 1, Molecular and Genetic Methods, ed. Keeney S. (Humana Press, Totowa, NJ), pp. $55-63$.

29. Miné-Hattab J, Rothstein R (2012) Increased chromosome mobility facilitates homology search during recombination. Nature cell biology 14(5):510-517.

30. Weber SC, Spakowitz AJ, Theriot JA (2010) Bacterial Chromosomal Loci Move Subdiffusively through a Viscoelastic Cytoplasm. Physical Review Letters 104(23).

31. Weber SC, Thompson MA, Moerner WE, Spakowitz AJ, Theriot JA (2012) Analytical tools to distinguish the effects of localization error, confinement, and medium elasticity on the velocity autocorrelation function. Biophysical journal 102(11):2443-2450.

32. Weber SC, Spakowitz AJ, Theriot JA (2012) Nonthermal atp-dependent fluctuations contribute to the in vivo motion of chromosomal loci. Proceedings of the National Academy of Sciences 109(19):7338-7343.

33. Ghosh RP, et al. (2019) A fluorogenic array for temporally unlimited single-molecule tracking Nature chemical biology 15(4):401-409.

34. Hajjoul H, et al. (2013) High-throughput chromatin motion tracking in living yeast reveals the flexibility of the fiber throughout the genome. Genome Research 23(11):1829-1838.

35. Marshall WF, Fung JC (2016) Modeling meiotic chromosome pairing: Nuclear envelope attachment, telomere-led active random motion, and anomalous diffusion. Physical Biology 13(2):026003.

36. Marshall WF, Fung JC (2019) Modeling meiotic chromosome pairing: A tug of war between telomere forces and a pairing-based Brownian ratchet leads to increased pairing fidelity. Physical Biology 16(4):046005.

37. Doi M, Edwards SF, Edwards SF (1988) The theory of polymer dynamics. (oxford university press) Vol. 73.

38. Lee CY, et al. (2015) Mechanism and regulation of rapid telomere prophase movements in mouse meiotic chromosomes. Cell reports 11(4):551-563.

39. Conrad MN, et al. (2008) Rapid Telomere Movement in Meiotic Prophase Is Promoted By NDJ1, MPS3, and CSM4 and Is Modulated by Recombination. Cell 133(7):1175-1187.

40. Wang X, et al. (2008) Rapid telomere motions in live human cells analyzed by highly time resolved microscopy. Epigenetics \& Chromatin 1:4.

41. Chu FY, Haley SC, Zidovska A (2017) On the origin of shape fluctuations of the cell nucleus. Proceedings of the National Academy of Sciences 114(39):10338-10343.

42. Wilemski G, Fixman M (1974) Diffusion-controlled intrachain reactions of polymers. I Theory The Journal of Chemical Physics 60(3):866-877.

43. Chacón MR, Delivani P, Tolić IM (2016) Meiotic Nuclear Oscillations Are Necessary to Avoid Excessive Chromosome Associations. Cell Reports 17(6):1632-1645.

44. Vazquez J, Belmont AS, Sedat JW (2002) The dynamics of homologous chromosome pairing during male Drosophila meiosis. Current biology: CB 12(17):1473-1483.

45. Wynne DJ, Rog O, Carlton PM, Dernburg AF (2012) Dynein-dependent processive chromosome motions promote homologous pairing in C. elegans meiosis. The Journal of Cell Biology 196(1):47-64.

46. Lampo TJ, Stylianidou S, Backlund MP, Wiggins PA, Spakowitz AJ (2017) Cytoplasmic RNA-Protein Particles Exhibit Non-Gaussian Subdiffusive Behavior. Biophysical Journal 112(3):532-542.

47. Pan J, et al. (2011) A hierarchical combination of factors shapes the genome-wide topography of yeast meiotic recombination initiation. Cell 144(5):719-731.

48. Mancera E, Bourgon R, Brozzi A, Huber W, Steinmetz LM (2008) High-resolution mapping of meiotic crossovers and non-crossovers in yeast. Nature 454(7203):479-485.

49. Qi J, et al. (2009) Characterization of meiotic crossovers and gene conversion by wholegenome sequencing in Saccharomyces cerevisiae. BMC Genomics 10(1):475.

50. Cooper TJ, Garcia V, Neale MJ (2016) Meiotic dsb patterning: A multifaceted process. Cell cycle 15(1):13-21.

51. Galupa R, Crocker J (2020) Enhancer-Promoter Communication: Thinking Outside the TAD. Trends in Genetics $0(0)$.

52. Doob JL (1942) TOPICS IN THE THEORY OF MARKOFF CHAINS. Trans. Amer. Math. Soc. 52:37-64.

53. Gillespie DT (1977) Exact stochastic simulation of coupled chemical reactions. The Journal of Physical Chemistry 81(25):2340-2361.

54. Lewis EB (1954) The Theory and Application of a New Method of Detecting Chromosomal Rearrangements in Drosophila melanogaster. The American Naturalist 88(841):225-239.

55. Loidl J (1990) The initiation of meiotic chromosome pairing: The cytological view. Genome 33(6):759-778.

56. Dresser ME (2009) Time-Lapse Fluorescence Microscopy of Saccharomyces cerevisiae in Meiosis in Meiosis, ed. Keeney S. (Humana Press, Totowa, NJ) Vol. 558, pp. 65-79.

57. Thomann D, Rines DR, Sorger PK, Danuser G (2002) Automatic fluorescent tag detection in $3 \mathrm{D}$ with super-resolution: Application to the analysis of chromosome movement. Journal of Microscopy 208(1):49-64.

58. Beltran B, MachPherson Q, Spakowitz AJ (2020) Waiting Times from Finite Trajectories. In Preparation 